\title{
Stress in Thin Wall Structures Made by Layer Additive Manufacturing
}

\author{
Vladimir Luzin ${ }^{1,{ }^{*}}$, Nicholas Hoye ${ }^{2, b}$ \\ ${ }^{1}$ Australian Nuclear Science and Technology Organisation, Locked Bag 2001, Kirrawee DC, \\ NSW, 2232 Australia \\ ${ }^{2}$ Faculty of Engineering and Information Sciences, University of Wollongong, NSW 2522 \\ Australia \\ avvl@ansto.gov.au, ${ }^{b}$ nicholas.hoye@gmail.com
}

\section{Keywords: Residual Stress, Additive Manufacturing, Titanium}

\begin{abstract}
Manufacturing of thin wall structures is one of the main applications of additive manufacturing, where it has significant advantages over traditional milling and machining techniques or welded analogues. Such thin walled structures are common in structural aerospace components, and are also frequently made from titanium alloys. For such large-scale components, layer deposition strategy is more advantageous rather than a pixel-wise deposition approach due to the demand for high productivity and size requirements. Several techniques can be used to produce layer-wise buildups, including laser-powered Direct Metal Deposition (DMD) process or gas tungsten arc welding (GTAW). Although, in the general case of arbitrary thin wall structures the stress distribution is complex, for some simple geometries, the stress state is simple and can be well characterized within a model by a single parameter representing a layer deposition stress in the steady-state regime. The model calculations were verified by experimental results on a thin-walled sample component that was manufactured from Ti-6Al-4V by GTAW with the residual stresses measured using KOWARI neutron strain scanner at the OPAL research reactor (ANSTO).
\end{abstract}

\section{Introduction}

Titanium based alloys are very widely used in aerospace industry due to their high specific strength, fatigue properties and excellent corrosion/oxidation resistance [1], and frequently need to be shaped into thin-wall structures, such as wing ribs and spars, various structural elements, gear boxes, etc. Traditional metal forming (e.g. rolling, extrusion) and machining methods are difficult, labour/cost intensive [2] and frequently extremely wasteful, especially when thin-wall structures are to be fabricated, resulting in unacceptably high buy-to-fly ratios. In comparison, additive manufacturing (AM) of titanium components seems to be the most attractive manufacturing technology, dramatically improving manufacturing costs and reducing waste to minimum with almost no limitation on the component shape. While some components for aerospace applications are small scale $(<300 \mathrm{~mm})$ and can be readily manufactured by powder-bed techniques such as Selective Laser Melting (SLM) or Electron Beam Melting (EBM), large-scale major airplane components present challenges due to size limitations of the build chamber. In this case, other techniques such as blownpowder based Direct Metal Deposition (DMD) or wire-fed based Wire-Arc Additive Manufacturing (WAAM) are used. The latter is also often called Wire-Arc Additive Layer Manufacturing (WAALM) since fabrication route involves multiple-pass deposition to build wall-like structures in a layer-by-layer manner to produce the 'near net shape' profile. Development of these techniques into industrial scale for high quality production of engineering components from titanium alloys in an economically efficient way is a general challenge for AM technology at present.

Although many technological process parameters such as deposition energy and speed, feedstock deposition rate, built-up trajectory, clamping system, resulting microstructure and defect structure are 
to be considered when optimising product quality and production costs, the residual stress is one of the most serious issues, being the core reason for significant bowing, bending and deflection, often resulting in compromised dimensional tolerances. For experimental studies, a single wall structure (T-shape sample) of a constant thickness and height, built on a rectangular base plate appears to be a standard choice [3-6]. Depending on the material, exact dimensions of the build-up and the process, the deflection of the back side of the base plate can vary greatly, but a typical reported deflection of the base plate in case of Ti-6Al-4V is $14 \mathrm{~mm}$ per $1000 \mathrm{~mm}$ longitudinal base length [6]. In addition to causing distortion, residual stresses may have detrimental effects on mechanical properties, especially fatigue behaviour, thereby degrading the performance of the component in service.

Considering AM process of a T-shape sample, two distinct steps or episodes in the overall stress formation can be isolated. When first layer is deposited a stress distribution in the base plate is created which is essentially very similar in nature and stress distribution to a single-bead welding path [5]. Although second and third passes can modify the stress distribution created by the first pass, all consecutive passes outstanding several $\mathrm{mm}$ from the base plate do not affect the base stress distribution. Instead they build-up stress in a different manner dictated by the geometry of the thinwall structure. If the wall thickness is much smaller than the wall height and length, the zero plane stress condition is applicable with transverse stress (through thickness) being equal to zero.

The aim of the current study is to investigate residual stress build-up in a T-shaped sample made from Ti-6Al-4V by WAALM in order to quantify residual stress process within an empirical model. One of the multiple possibilities is to consider different scenarios for clamping and its influence on the resultant residual stress. This quantification will allow certain conclusions to be drawn about the mechanism(s) of residual stress formation, hence allowing the prediction of residual stresses in samples of different dimensions and, possibly, to evaluate residual stress mitigation strategies.

\section{Sample production, process and materials}

WAALM, as an arc-based deposition process can be realised in several ways, it can utilize either the gas metal arc welding (GMAW) or the gas tungsten arc welding (GTAW). The latter version has been developed in the University of Wollongong as a practical AM method with high deposition rate enabling production of large components [7]. In this process, build-up of a three-dimensional near-net shape freeform is achieved through deposition of a single row of successive weld beads onto a substrate to produce a component in a layer-wise manner. The process utilizes robotic automation that can be

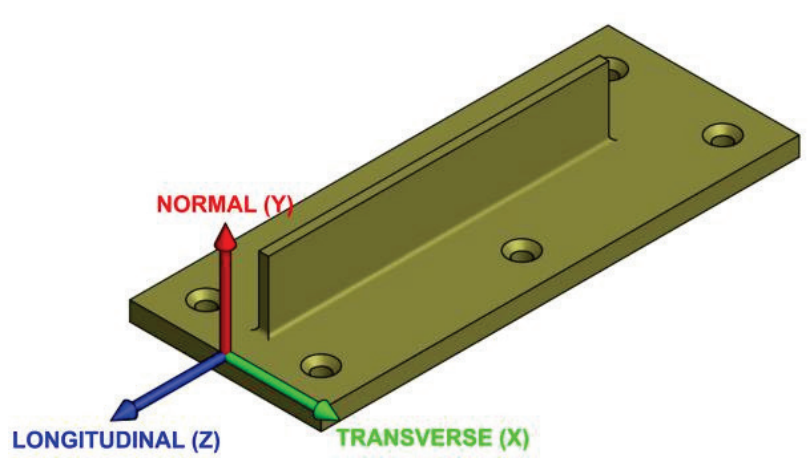

Fig. 1. Design of the thin-wall structure produced by WAALM with indication of the principal directions. programmed to suit the design of the future component [8].

Using the reported WAALM, a thin-wall of Ti-6Al-4V was built to the full length along the centre line of a Ti-6Al-4V base plate (L250xW100xT12, mm). The resulting build-up thin wall was approximately $8 \mathrm{~mm}$ in thnessi and $40 \mathrm{~mm}$ in height. The as-deposited was further machined via conventional milling to a wall of high precision dimensions with $5 \mathrm{~mm}$ thickness, $36 \mathrm{~mm}$ height and $178 \mathrm{~mm}$ length. The final shape of the component is shown in Fig. 1.

Parameters of deposition GTA welding process have been already reported [9]. The base plate, attached to a linear actuator, was moved with a travel speed of $150 \mathrm{~mm} / \mathrm{min}$, while the welding torch and wire feed were held stationary. Deposition was conducted using a current-controlled power source operating at a steady state current of 110 A and giving an average arc energy of $485 \mathrm{~J} / \mathrm{mm}$. Filler wire of $1.0 \mathrm{~mm}$ diameter was fed at a rate of $2000 \mathrm{~mm} / \mathrm{min}$ to provide a specific energy input of approximately $10 \mathrm{~kJ}$ per gram of deposited material. Another sample was produced at conditions 
of $20 \mathrm{~kJ}$ per gram of deposited material by reducing the travel speed by a factor of two Both, the base plate and the filler wire materials were commercially sourced and produced to ASTM B265 and B863 standards respectively.

\section{Neutron diffraction experiment and data analysis}

Neutron residual stress measurements of the wall-structure were performed on the KOWARI neutron diffractometer at OPAL research reactor at ANSTO [10]. For the measurement the Ti(103) reflection was used at $90^{\circ}$-geometry employing a neutron wavelength of approximately $\lambda=1.7 \AA$. Three principal directions were measured, the normal, transverse and longitudinal across the wall height (from base plate to the top of the wall) in the middle portion of the $250 \mathrm{~mm}$ long sample with variable density of the measurement point $(1 \mathrm{~mm}$ close to the base plate and $2 \mathrm{~mm}$ away from the base plate). More than 20 experimental points along the wall height were obtained by scanning.

To use efficiently sample geometry, a gauge volume with size of $2 \times 2 \times 20 \mathrm{~mm}^{3}$ was used for measurements of the normal and transverse strain components, while this gauge volume was reduced to $2 \times 2 \times 2 \mathrm{~mm}^{3}$ when the longitudinal component was measured. To acquire better grain statistics, the gauge volume was moved during measurements of the longitudinal component to cover equivalent volume of $2 \times 2 \times 20 \mathrm{~mm}^{3}$. For the given experimental conditions, an average accuracy of $\sim 100 \mu$ strain was achieved providing stress accuracy of $\sim 20 \mathrm{MPa}$ in terms of calculated stresses (errors only due to the neutron counting statistics).

With respect to determining the sample $d_{0}$, the more standard approach of cutting small coupons that can be assumed stress free proved unreliable due to poor statistics associated with the comparatively large prior $\beta$-Ti grain size. As only the stress distribution in the thin wall section was of interest, an alternative approach was employed where the through thickness stress was assumed to be zero in the thin wall section. This assumption of a plane stress condition is considered valid due to the $5 \mathrm{~mm}$ wall thickness being of similar size to the $\sim 3 \mathrm{~mm}$ gauge volume (or spatial resolution), and was seen to give results with great accuracy. Based on this condition, three d-spacings for three directions could be resolved into two stress components (longitudinal and normal) and $\mathrm{d}_{0}$.

\section{Modelling of stress profile in a wall}

A simple model can be considered based on the established experimental facts that the transverse and normal components are insignificant in comparison to the dominant longitudinal component. The wall build-up process can be considered as a progressive deposition of the infinitesimal layers of material characterised by a single parameter, the deposition stress $\sigma_{\mathrm{d}}$, just as the progressive deposition of layers can be applied to describe stress formation in coatings [11]. Although a generalized model can deal with dissimilar materials of the wall and the base plate, for our particular purpose, we consider only similar materials for both (same Young's modulus and Poisson ratio).

While a newly deposited layer cools down, shrinks and acquire

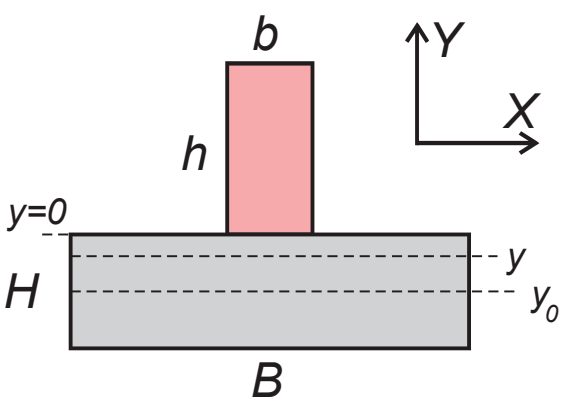

Fig. 2. Geometry and dimensions of the wall-on-base sample. some intrinsic quenching stress or deposition stress in general, the T-beam bending theory can be applied to find the resultant stress distribution required to satisfy the force balance and the moment balance [12]. The resultant stress distribution can be computed in few steps. The deposition stress $\sigma_{d}$, in a wall element, of width $b$ and height $h$, built on the base plate, of width $B$ and thickness $H$, causes the appearance of a pair of equal and opposite forces, $F$, (one in the wall, one in the base)

$$
F=\sigma_{d} \frac{b h \cdot B H}{b h+B H} \quad \text { and a moment, } M, \quad M=F \frac{(h+H)}{2} .
$$

The induced stress distribution in the wall is according to

$$
\sigma(y)=\frac{F}{b h}-\frac{M}{I}(y-\bar{y})
$$


where position of the neutral axis $\bar{y}$ and area moment of inertia $I$ can be calculated for the (composite) beam as follows

$$
\begin{aligned}
& \bar{y}=\frac{b h \frac{h}{2}-B H \frac{H}{2}}{b h+B H}, \\
& I=\frac{B H^{3}}{12}+B H\left(\frac{H}{2}+\bar{y}\right)^{2}+\frac{b h^{3}}{12}+b h\left(\frac{h}{2}-\bar{y}\right)^{2} .
\end{aligned}
$$

The same forces also produce curvature, $\kappa$,

$$
\kappa=\frac{M}{I E}=\frac{\sigma_{d}}{E} \cdot(\text { geometrical factor }),
$$

which is proportional to the deposition stress.

Using the above formulae, the two extreme scenarios can be considered that can be called, "fully constrained" and "fully free" base plate. In the former case, the full wall height is first build up and then the mechanical equilibrium is achieved, as would be expected when the thin-walled section is released from the constrains of the base plate. In the latter case, the equilibrium is found after each episode of the infinitesimal layer deposition, simulating continuous sample curvature change due to the stress build-up.

\section{Results}

The stress distributions of two stress components and two analysed samples are shown in Fig. 3. Although no assumption was made for the normal component, as it can be seen, it is very close to zero for both samples. Therefore, it can be concluded that not only the transverse component is zero, but also normal, what makes stress state very simple with only longitudinal component.

Although the specific energy input of two samples is different, there is no clear indication about correlation between stress and heat input. The only distinct feature of the high specific energy input sample, $20 \mathrm{~kJ} / \mathrm{g}$, is larger statistical oscillations most evidently related to the coarser-grained microstructure.
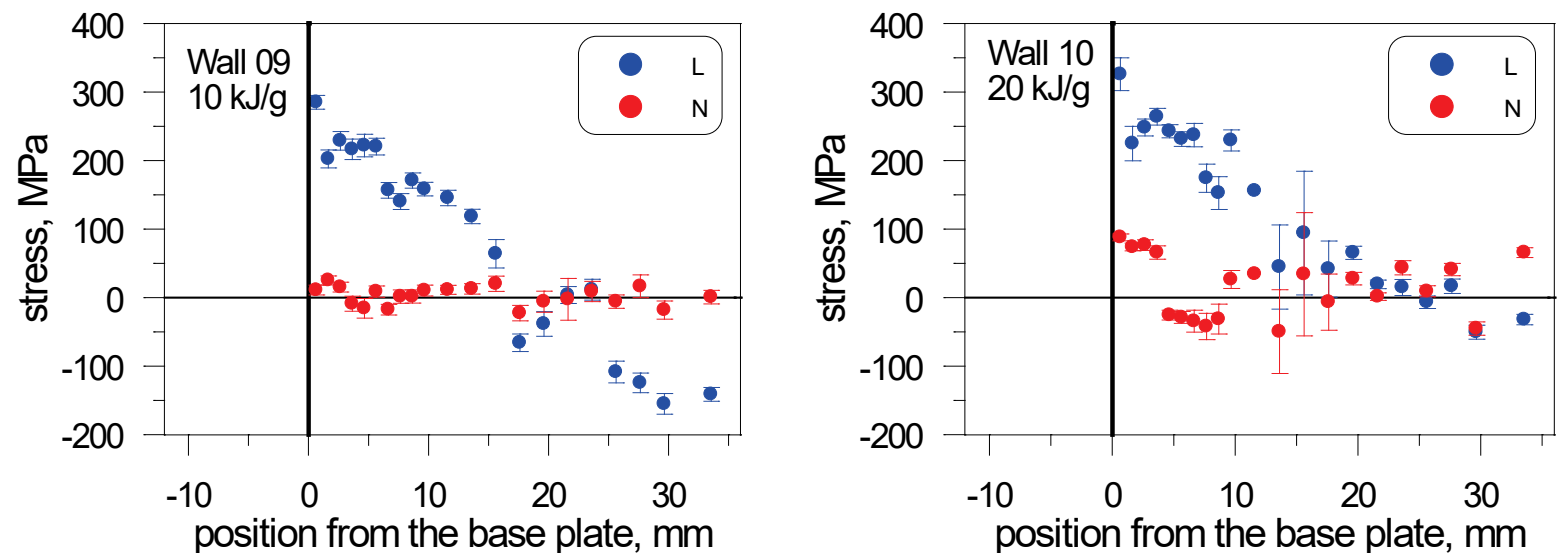

Fig. 3. Residual stress analysis for the samples of two heat input, $10 \mathrm{~kJ} / \mathrm{g}$ and $20 \mathrm{~kJ} / \mathrm{g}$.

The neutron diffraction stress distributions in the walls were analysed with the modelling approach discussed above to produce a fit to the experimental dataset. Two scenarios, fully constrained sample and fully free, were considered and their comparison is shown in Fig. 4 together with the experimental results from the both samples. Clearly, the "fully constrained" sample assumption works the best and provides the linear trend of stress dependence exhibited in the experimental data, while "no constrain" sample obviously does not follow the trend and has a distinct non-linearity. In both cases, the deposition stress parameter was $400 \mathrm{MPa}$. 
The same approach was applied to other datasets taken from the published literature. In one case the Ti-6Al-4V alloy $6 \mathrm{~mm}$ thin wall was additively manufactured by a similar technique and measured using neutron diffraction [6]. In the other case, a stress analysis of a steel $5 \mathrm{~mm}$ thin wall on the steel substrate was carried out also with neutron diffraction [5]. The experimental data from these studies also agrees with "full constrain" profiles, as shown in Fig. 5. The corresponding deposition stress is $400 \mathrm{MPa}$ for the Ti-6Al-4V samples and $500 \mathrm{MPa}$ for the steel sample.

\section{Discussion}

Three Ti-6Al-4V samples, considered in terms of deposition model, demonstrate remarkably close outcome, $400 \mathrm{MPa}$ deposition stress. This result suggests that material properties (i.e. yield stress) are the most important factor for the stress formation. Although details of the deposition process such as deposition rate, travel speed, energy input, etc., might vary, the thermomechanical history of the deposited material seems to remain within a narrow envelop of physical parameters providing conditions for a stable and efficient build-up of the wall. Considering this, it is evident that the deposition stress is a result of quenching with accumulation first of very high stress, up to the yield point, followed by some partial annealing by subsequent passes.
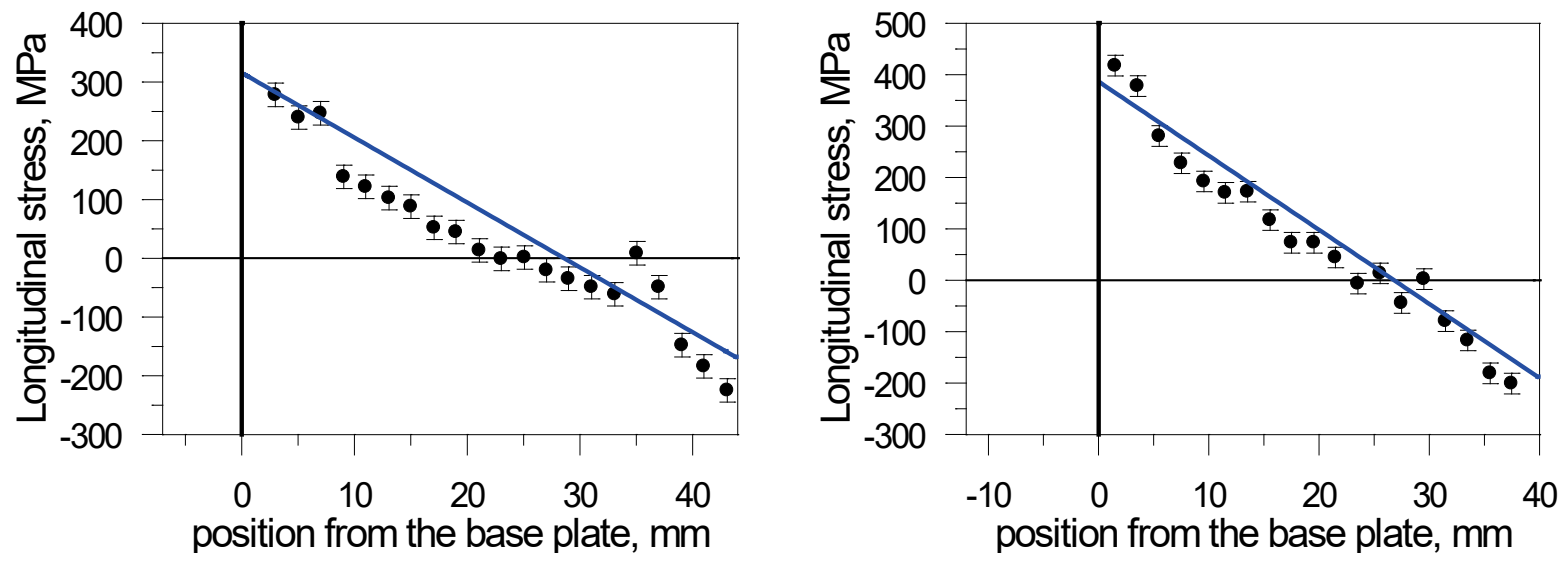

Fig. 5. Experimental residual stress distributions in walls two samples, a Ti-6Al-4V thin wall (left, adopted from [6]) and a steel thin wall (right, adopted from [5]). Experimental points are overlapped with simulated stress profiles (blue lines) calculated using "full constrain"

The stress build-up can be easily and successfully modelled within a simple model based on a beam bending theory and an assumption (and practical realisation) of the full constraint of the base plate. The model assumes very simple stress state with only the longitudinal component being nonzero and it is fully characterised by a single parameter, the deposition stress $\sigma_{d}$ or, equivalently, the curvature $\kappa$. The fact that the normal and transverse stresses can be accepted as zero for all practical purposes has a solid experimental corroboration in this study as well as in other studies [4,6]. While not every detail can be derived with the simple analytical model, it is clear that the main trend and magnitude of stress distribution in the wall can be reproduced. It is worth noting that this simplified 
modelling approach is most likely unable to accurately characterise the stress field at the joint point with the base plate, as well as at the very top layer of deposited material, which would have a different history to all other deposited layers.

The tensile deposition stress due to quenching, as high as $500 \mathrm{MPa}$, is potentially detrimental to mechanical properties and most certainly leads to significant curvature of the base plate. Some stress mitigation approaches have been attempted to reduce stresses [4], though the definition of the most practical and most efficient solution remains one of the challenges for additive manufacturing technologies.

\section{Summary}

In this study, an experimental and modelling approach were attempted to characterize residual stress distributions in a thin wall deposited by additive manufacturing (WAALM). It was demonstrated that the stress state may be characterised only by the longitudinal component and that the model can provide a general steady-state linear behaviour of the longitudinal stress distribution in the wall observed experimentally. Typically very high tensile deposition stresses are produced by WAALM, $400 \mathrm{MPa}$ for Ti-6Al-4V and $500 \mathrm{MPa}$, as in the reported examples, highlighting the requirement for stress mitigating strategies if component distortions during additive manufacturing are to be avoided.

\section{References}

[1] S. Seong, O. Younossi, B.W. Goldsmith, T. Lang, M. Neumann, Titanium: Industrial Base, Price Trends, and Technology Initiatives, RAND Project Air Force, 2009.

[2] J.P. Davim, Machining of Titanium Alloys, Springer Berlin Heidelberg, 2014. http://dx.doi.org/10.1007/978-3-662-43902-9

[3] B. Baufeld, O.V.d. Biest, R. Gault, Additive manufacturing of Ti-6Al-4V components by shaped metal deposition: Microstructure and mechanical properties, Mat. Des., 31, Supplement 1 (2010) S106-S111. http://dx.doi.org/10.1016/j.matdes.2009.11.032

[4] P.A. Colegrove, H.E. Coules, J. Fairman, F. Martina, T. Kashoob, H. Mamash, L.D. Cozzolino, Microstructure and residual stress improvement in wire and arc additively manufactured parts through high-pressure rolling, J. Mater. Process. Technol., 213 (2013) 1782-1791. http://dx.doi.org/10.1016/j.jmatprotec.2013.04.012

[5] J. Ding, P. Colegrove, J. Mehnen, S. Ganguly, P.M. Sequeira Almeida, F. Wang, S. Williams, Thermo-mechanical analysis of Wire and Arc Additive Layer Manufacturing process on large multilayer parts, Computational Materials Science, $50 \quad$ (2011) 3315-3322. http://dx.doi.org/10.1016/j.commatsci.2011.06.023

[6] B.A. Szost, S. Terzi, F. Martina, D. Boisselier, A. Prytuliak, T. Pirling, M. Hofmann, D.J. Jarvis, A comparative study of additive manufacturing techniques: Residual stress and microstructural analysis of CLAD and WAAM printed Ti-6Al-4V components, Mat. Des., 89 (2016) 559-567. http://dx.doi.org/10.1016/j.matdes.2015.09.115

[7] D. Ding, Z. Pan, D. Cuiuri, H. Li, A multi-bead overlapping model for robotic wire and arc additive manufacturing (WAAM), Robotics and Computer-Integrated Manufacturing, 31 (2015) 101110. http://dx.doi.org/10.1016/j.rcim.2014.08.008

[8] D. Ding, C. Shen, Z. Pan, D. Cuiuri, H. Li, N. Larkin, S. van Duin, Towards an automated robotic arc-welding-based additive manufacturing system from CAD to finished part, Computer-Aided Design, 73 (2016) 66-75. http://dx.doi.org/10.1016/j.cad.2015.12.003

[9] N. Hoye, H. Li, D. Cuiuri, A. Paradowska, Measurement of residual stresses in titanium aerospace components formed via additive manufacturing, Mater. Sci. Forum, 777 (2014) 124-129.

[10] O. Kirstein, V. Luzin, U. Garbe, The Strain-Scanning Diffractometer Kowari, Neutron News, 20 (2009) 34-36.

[11] Y.C. Tsui, T.W. Clyne, An analytical model for predicting residual stresses in progressively deposited coatings .1. Planar geometry, Thin Solid Films, 306 (1997) 23-33. http://dx.doi.org/10.1016/S0040-6090(97)00199-5

[12] S. Timoshenko, J.M. Gere, Mechanics of materials, Van Nostrand Reinhold Co., 1972. 